\title{
Network Pharmacology Integrated Molecular Docking Reveals the Mechanism of Zhongfeng Xingnao Formula against Intracerebral Hemorrhage
}

\section{Can Wan}

Guangzhou University of Traditional Chinese Medicine: Guangzhou University of Chinese Medicine

\section{Ziyi Zhou}

Guangdong Provincial Hospital of Traditional Chinese Medicine

\section{Yun Lu}

Chengdu University of Traditional Chinese Medicine Affiliated Hospital

\section{Guangyao Zhang}

Guangdong University of Technology

\section{Yefeng Cai}

Guangdong Provincial Hospital of Traditional Chinese Medicine

Jianwen Guo ( $\Delta 306247680 @ q q . c o m$ )

Guangdong Provincial Hospital of Traditional Chinese Medicine https://orcid.org/0000-0003-00215266

\section{Research}

Keywords: Network pharmacology, Molecular docking, Zhongfeng Xingnao Formula, Intracerebral hemorrhage, Effector mechanism

Posted Date: December 4th, 2020

DOl: https://doi.org/10.21203/rs.3.rs-117791/v1

License: (a) (1) This work is licensed under a Creative Commons Attribution 4.0 International License. Read Full License 


\section{Abstract}

Background: Previous studies have shown that Zhongfeng Xingnao Formula (ZXF) can effectively reduce the mortality of intracerebral hemorrhage $(\mathrm{ICH})$, but the underlying mechanism of the treatment remained still unexplored. This study aimed to expound the potential mechanism of ZXF in the treatment of ICH through network pharmacology and molecular docking.

Methods: The putative targets of ZXF were obtained from the TCMSP and Uniprot database, while the potential targets of ICH received from Drugbank, Genecards and OMIM database. Then through the Venn 2.1, the overlapping targets of disease and drug were gotten for the further study. The GO and KEGG enrichment analyses were performed by $\mathrm{R}$ version 4.0.2 software so that the signaling pathway was acquired to the subsequent analysis. Cytoscape was used to construct the drug-compound-targetpathway network and String was utilized for the protein-protein interaction network. What's more, the interaction between compound and target was verified by the AutoDockTools and Autodock Vina.

Results: There were a total of 166 ZXF-related targets and $1258 \mathrm{ICH}$-related targets obtained from the public databases. And 87 potential targets were both related to drug and disease. The GO enrichment analysis mainly involved receptor ligand activity, signaling receptor activator activity, and cytokine receptor binding, while the signaling pathway, such as Fluid shear stress and atherosclerosis, AGE-RAGE signaling pathway in diabetic complications, PI3K-Akt signaling pathway, were significantly enriched in the KEGG enrichment analysis. The molecular docking elucidated that the aloe-emodin, beta-sitosterol, quercetin could bound well to the top five targets sorted by degree value.

Conclusions: ZXF treated ICH through multiple compounds, multiple targets, and multiple pathways. The underlying mechanism of the treatment may be promoting angiogenesis, anti-inflammatory, antioxidative stress, and reversing atherosclerosis, which is of great significance for the treatment of ICH.

\section{Introduction}

Intracerebral hemorrhage $(\mathrm{ICH})$ is a form of stroke with high morbidity, accounting for about $14.9 \%$ and $10 \%$ of all strokes in China and the United States respectively [1,2]. What's worse, ICH is also a devastating disease with awfully high rate of disability and mortality [3]. However, there is almost no optimal treatment for ICH until now in the modern medicine field [4]. The Antihypertensive Treatment of Acute Cerebral Hemorrhage $\triangle($ ATACH-2) trial showed that intensive blood-pressure lowering cannot lead to a decline in mortality and disability [5]. And in the Intracerebral Haemorrhage Deferoxamine (i-DEF) trial, there was no significant evidence for the improvement in the 90-day good outcome [6]. In addition, the Tranexamic acid for hyperacute primary IntraCerebral Haemorrhage (TICH-2) trial indicated that there was no significant difference in 90-day functional status between placebo-treated and tranexamic acidtreated patients [7]. Therefore, it is of great clinical importance to discover therapeutic drugs and formulations for the treatment of $\mathrm{ICH}$. 
In the field of traditional Chinese medicine (TCM), more and more attention was fixed on the therapy of stroke, especially hemorrhagic stroke [8]. Song et al found that Naoxueshu oral liquid can significantly reduce the inflammation and edema of $\mathrm{ICH}$ [9]. And $\mathrm{Li}$ et al. reported that tsRNAs may be the potential targets for Buyang Huanwu Decoction against ICH [10]. Moreover, the treatment of ICH by ZXF has been proven to effectively reduce the mortality, promote the absorption of hematoma, and improve the neurological deficits [11]. The Zhongfeng Xingnao Formula (ZXF), a widely acclaimed anti-ICH Chinese patent medicine, is composed of four Chinese herbs, such as Rhubarb (Dahuang in Chinese, DH), Panax Notoginseng (Sanqi in Chinese, SQ), Chuanxiong Rhizoma (Chuanxiong in Chinese, CX), Red Ginseng (Hongshen in Chinese, HS). It has the effects of replenishing qi, activating blood, resolving stasis, and stopping bleeding. Nevertheless, the underlying mechanism of ZXF in the treatment of ICH still remained unclear.

Network pharmacology is an emerging discipline combined with pharmacology and bioinformatics [12], which was first proposed by Hopkins in 2007 [13]. It believes that drugs exert effects through the interaction between multiple components and multiple targets, which coincides with the characteristics of traditional Chinese medicine [14]. Molecular docking is a widely used structure-based silico method, which is able to get the binding energy and binding mode of receptors and ligands through scoring function [15]. In this study, the network pharmacology and molecular docking were used to explore the active compounds of ZXF, the putative targets of ZXF against ICH, and the potential mechanism of ZXF in the treatment of $\mathrm{ICH}$.

\section{Materials And Methods}

\section{Collection and screening of active compounds}

The compounds in ZXF were collected in Traditional Chinese Medicine Systems Pharmacology Database and Analysis Platform (TCMSP) database [16](https://tcmspw.com/tcmsp.php) whose search terms were as follows: Dahuang (DH, rhubarb in English), Sanqi (SQ, sanchi in English), Chuanxiong (CX, szechwan lovage rhizome in English), Hongshen (HS, red ginseng in English). TCMSP database is an important pharmacology platform of Chinese herbs that provides critical data about pharmacokinetic properties, such as oral bioavailability (OB) [17], drug-likeness (DL) [18], intestinal epithelial permeability (IEP), blood-brain-barrier (BBB) and etc. Therefore, the compounds with $\mathrm{OB} \geq 30 \%$ and $\mathrm{DL} \geq 0.18$ simultaneously in the TCMSP database were screened as active compounds for the further study.

\section{Prediction of drug-related targets.}

The validated target proteins of the active compounds were further predicted with the TCMSP database. And then the UniProt database (https://www.uniprot.org/) was utilized to convert the name of target protein into UniProt ID by the UniProt Knowledge Base (UniProtKB), with the organism limited to "Homo 
sapiens" and the active compounds without UniProt ID eliminated. Ultimately the official Gene symbols were received from the abovementioned steps [19].

\section{Identification of disease-related targets.}

The OMIM database (https://www.omim.org/) [20], GeneCards database (https://www.genecards.org/) [21], DrugBank database (https://go.drugbank.com/) [22]were used to search for diseases-related targets with the keywords "Cerebral Hemorrhage", "Intracerebral Hemorrhage", and "Intracranial Hemorrhage". And then the search results were summarized in an excel table and the duplicate data was removed from the table. Eventually the disease-related targets would be included in the subsequent analysis.

\section{Acquisition of ZXF targets associated with ICH.}

The drug-related targets and disease-related targets were both imported into Venn 2.1 (https://bioinfogp.cnb.csic.es/tools/venny/index.html), in which the Venn diagram was constructed. In addition, the intersection of the two kinds of targets was obtained from the website, which were the potential targets of ZXF against ICH.

\section{Analysis of function and pathway Enrichment.}

$R$ version 4.0.2 software was used for Gene Ontology (GO) biological function enrichment analysis and Kyoto Encyclopedia of Genes and Genomes (KEGG) metabolic pathway enrichment analysis [23, 24]. The threshold was set to P.adjust $₫ 0.05$. Through the $\mathrm{GO}$ enrichment analysis, the biological process (BP) terms, cell component (CC) terms, and molecular function (MF) terms which potential targets most highly enriched in could be obtained [25]. The top 10 terms sorted by gene ratio would be presented in the form of bubble plots. In addition, the signaling pathways for ZXF against ICH can be received from KEGG enrichment analysis [26]. The bar plots would be used to demonstrate the top 20 pathways sorted by $P$ value.

\section{Construction of drug-compound-target-pathway network}

The Cytoscape 3.7.1 software was used to construct the drug-component-target-pathway network [27]. Nodes with different colors and shapes were used to represent drugs, compounds, potential targets, and signaling pathways respectively. The edges were used to connect the drug-compound, compound-target, and target-pathway. The top 20 signaling pathways were sorted by gene ratio and all potential targets were included in the network.

\section{Establishment of protein-protein interaction}


The potential targets were imported into the String database (https://string-db.org/) with the organism set to "Home sapiens", the minimum required interaction score limited to "Highest confidence 0.400 ", and hiding the disconnected nodes [28]. Then the protein-protein interaction (PPI) network was established.

The PPI network was input to the Cytoscape 3.7.1 software, whose NetworkAnalyser function was used to calculate the degree value of the nodes. The degree value, one of the topological parameters, can reflect the significance of the node. Next, the Cytoscape software was used to visualize the degree value of the potential targets. The nodes with larger degree value were with larger size and darker color in the figure. In addition, the top 10 potential targets sorted by degree value, namely the core genes, also used the above method to construct a PPI network.

\section{Verification by molecular docking.}

Molecular docking is a widely used technology based on the theory of lock and key, revealing how the proteins and ligands interact with each other [29]. Therefore, the connections between the core targets and the active compounds were verified by molecular docking in our study. Firstly, the PDB format files of core targets were downloaded from the RSCB PDB database (https://www.rcsb.org/ ) [30], while the mol2 format files of the active compounds were downloaded from the TCMSP database. Secondly, the AutoDockTools 1.5.6 was used to pretreat the core targets and active compounds, including deleting water, adding hydrogens and computing charges. Also, the grid box was created by the AutoDockTools with coordinates and size of the box recorded [31]. Thirdly, the Autodock Vina 1.1.2 was used for docking operation to get the binding mode and binding affinity [32]. Last but not least, the PyMOL 2.4.0 was used to visualize the results of molecular docking to check the interaction between core targets and active compounds [33].

\section{Results}

\section{Active compounds in ZXF.}

After the collection and screening in the TCMSP database, a total of 16 active compounds were obtained in ZXF, including 6 Rhubarb, 3 Red Ginseng, 7 Panax Notoginseng, and 4 Chuanxiong Rhizoma. Three of sixteen active compounds were shared by two or three Chinese herbs, such as beta-sitosterol, ginsenoside rh2, Mandenol. The detailed information was shown in Table 1. 
Table 1

Information on the active compounds in Zhongfeng Xingnao Formula

\begin{tabular}{|llllll|}
\hline ID & Mol. ID & Chemical name & OB(\%) & DL & Herbs \\
\hline A & MOL000358 & beta-sitosterol & 36.91 & 0.75 & HX, SQ, DH \\
\hline B & MOL005344 & ginsenoside rh2 & 36.32 & 0.56 & HS, SQ \\
\hline CX1 & MOL002135 & Myricanone & 40.6 & 0.51 & CX \\
\hline CX2 & MOL002157 & wallichilide & 42.31 & 0.71 & CX \\
\hline CX3 & MOL000359 & Sitosterol & 36.91 & 0.75 & CX \\
\hline DH1 & MOL002235 & EUPATIN & 50.8 & 0.41 & DH \\
\hline DH2 & MOL002268 & Rhein & 47.07 & 0.28 & DH \\
\hline DH3 & MOL002281 & Toralactone & 46.46 & 0.24 & DH \\
\hline DH4 & MOL002297 & Daucosterol_qt & 35.89 & 0.7 & DH \\
\hline DH5 & MOL000471 & aloe-emodin & 83.38 & 0.24 & DH \\
\hline HS1 & MOL002032 & DNOP & 40.59 & 0.4 & HS \\
\hline SQ1 & MOL001792 & DFV & 32.76 & 0.18 & SQ \\
\hline SQ2 & MOL002879 & Diop & 43.59 & 0.39 & SQ \\
\hline SQ3 & MOL000449 & Stigmasterol & 43.83 & 0.76 & SQ \\
\hline SQ4 & MOL000098 & Quercetin & 46.43 & 0.28 & SQ \\
\hline
\end{tabular}

\section{Putative targets of ZXF.}

A total of 47 potential targets of Rhubarb, 36 potential targets of Red Ginseng, 154 potential targets of Panax Notoginseng, and 23 potential targets of Chuanxiong Rhizoma were obtained from the prediction of the TCMSP database and the conversion of the UniProt database. Among them, 32 potential targets were shared by two or more active compounds. Consequently there were 166 potential targets of ZXF in total for the following study.

\section{Potential targets of ICH.}

By searching the above databases, 36 potential targets were from OMIM database, while 1215 potential targets were from GeneCards database, and 24 potential targets were from DrugBank database. After 
screening, sorting, checking, deduplicating, and unifying the search results, a total of 1258 potential targets related to $\mathrm{ICH}$ were collected.

\section{Overlapping targets of ZXF and ICH.}

In the Venn diagram, there were 166 ZXF-related targets, $1258 \mathrm{ICH}$-related targets and 87 overlapping targets in total. It was worth mentioning that the 87 overlapping targets may be the potential targets of ZXF against ICH, which may play a critical role in the treatment of ICH (Fig. 2 and Table 2). 
Table 2

Potential targets of Zhongfeng Xingnao Formula against intracerebral hemorrhage

\begin{tabular}{|c|c|c|c|c|c|c|c|c|c|c|}
\hline NO. & Gene & & No. & Gene & & No. & Gene & & No. & Gene \\
\hline 1 & MMP2 & & 23 & CHRM3 & & 45 & RUNX2 & & 67 & IL2 \\
\hline 2 & PLAU & & 24 & IGFBP3 & & 46 & PGR & & 68 & ERBB3 \\
\hline 3 & Nos2 & & 25 & PTGS1 & & 47 & CHRM2 & & 69 & ERBB2 \\
\hline 4 & CCNB1 & & 26 & F3 & & 48 & ODC1 & & 70 & FOS \\
\hline 5 & BIRC5 & & 27 & JUN & & 49 & CXCL10 & & 71 & IFNG \\
\hline 6 & PON1 & & 28 & ESR2 & & 50 & SERPINE1 & & 72 & ICAM1 \\
\hline 7 & CHEK1 & & 29 & CD40LG & & 51 & VCAM1 & & 73 & BCL2L1 \\
\hline 8 & F7 & & 30 & RASSF1 & & 52 & TP53 & & 74 & CHEK2 \\
\hline 9 & CASP8 & 31 & & CYP3A4 & 53 & & CASP9 & 75 & & RELA \\
\hline 10 & CHRM1 & 32 & & CYP1B1 & 54 & & CDKN1A & 76 & & HIF1A \\
\hline 11 & PPARG & 33 & & PRSS1 & 55 & & RASA1 & 77 & & MAP2 \\
\hline 12 & CRP & 34 & & MMP9 & 56 & & AKT1 & 78 & & NOS3 \\
\hline 13 & GSTP1 & 35 & & TOP1 & 57 & & IL1B & 79 & & CASP3 \\
\hline 14 & CCL2 & 36 & & STAT1 & 58 & & IL6 & 80 & & ADRA1B \\
\hline 15 & EGF & 37 & & IL1A & 59 & & MPO & 81 & & NFE2L2 \\
\hline 16 & CXCL8 & 38 & & ADRA1A & 60 & & SELE & 82 & & ADCYAP1 \\
\hline 17 & KDR & 39 & & HMOX1 & 61 & & MMP3 & 83 & & PTGS2 \\
\hline 18 & PCNA & 40 & & CASP1 & 62 & & SPP1 & 84 & & MAPK14 \\
\hline 19 & THBD & 41 & & NR3C1 & 63 & & CHRM4 & 85 & & PLAT \\
\hline 20 & IL 10 & 42 & & MAPK1 & 64 & & IGF2 & 86 & & MYC \\
\hline 21 & CCND1 & 43 & & ESR1 & 65 & & VEGFA & 87 & & RB1 \\
\hline 22 & TNF & 44 & & EGFR & 66 & & GSK3B & & & \\
\hline
\end{tabular}

\section{GO and KEGG Enrichment Analysis.}

In the GO enrichment analysis, 87 potential targets were significantly enriched in $1782 \mathrm{BP}$ terms, $41 \mathrm{CC}$ terms, $112 \mathrm{MF}$ terms (P.adjust $\mathbb{0}$ 0.05). The terms were sorted in descending order from top to bottom by the gene ratio. As was shown in Fig. 3 , receptor ligand activity (P.adjust $=1.02 \mathrm{E}^{-07}$ ), signaling receptor 
activator activity (P.adjust $=1.02 \mathrm{E}^{-07}$ ), and cytokine receptor binding (P.adjust $\left.=2.65 \mathrm{E}^{-09}\right)$ were three most highly enriched GOMF terms. Among the $41 \mathrm{GOCC}$ terms, membrane raft (P.adjust $=1.34 \mathrm{E}^{-07}$ ), membrane microdomain (P.adjust $=1.34 \mathrm{E}^{-07}$ ), and membrane region (P.adjust $=1.40 \mathrm{E}^{-07}$ ) were three most closely related to cell component of $\mathrm{ICH}$. In terms of biological processes, potential targets concentrated more in response to lipopolysaccharide (P.adjust $\left.=5.03 \mathrm{E}^{-23}\right)$, response to molecule of bacterial origin (P.adjust $\left.=7.15 \mathrm{E}^{-23}\right)$ and cellular response to drug $\left(\right.$ P.adjust $\left.=4.80 \mathrm{E}^{-19}\right)$.

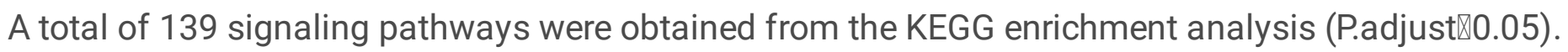
Among them, Fluid shear stress and atherosclerosis, AGE-RAGE signaling pathway in diabetic complications, PI3K-Akt signaling pathway, Kaposi sarcoma-associated herpesvirus infection, Human cytomegalovirus infection pathway were the most relevant pathway to ZXF against ICH (Fig. 3 and Table 3). 
Table 3

Information of signaling pathway obtained from KEGG enrichment analysis

\begin{tabular}{|llll|}
\hline ID & Description & p.adjust & Count \\
\hline hsa05418 & Fluid shear stress and atherosclerosis & $1.25 \mathrm{E}-21$ & 24 \\
\hline hsa04933 & AGE-RAGE signaling pathway in diabetic complications & $2.04 \mathrm{E}-23$ & 23 \\
\hline hsa04151 & Pl3K-Akt signaling pathway & $4.9 \mathrm{E}-12$ & 23 \\
\hline hsa05167 & Kaposi sarcoma-associated herpesvirus infection & $4.41 \mathrm{E}-16$ & 22 \\
\hline hsa05163 & Human cytomegalovirus infection & $9.97 \mathrm{E}-14$ & 21 \\
\hline hsa05161 & Hepatitis B & $2.52 \mathrm{E}-15$ & 20 \\
\hline hsa05205 & Proteoglycans in cancer & $1.91 \mathrm{E}-13$ & 20 \\
\hline hsa04010 & MAPK signaling pathway & $6.47 \mathrm{E}-11$ & 20 \\
\hline hsa04668 & TNF signaling pathway & $5.65 \mathrm{E}-17$ & 19 \\
\hline hsa04657 & IL-17 signaling pathway & $5.65 \mathrm{E}-17$ & 18 \\
\hline hsa05160 & Hepatitis C & $2.66 \mathrm{E}-13$ & 18 \\
\hline hsa05164 & Influenza A & $9.06 \mathrm{E}-13$ & 18 \\
\hline hsa05215 & Prostate cancer & $1.73 \mathrm{E}-15$ & 17 \\
\hline hsa05142 & Chagas disease & $3.29 \mathrm{E}-15$ & 17 \\
\hline hsa04218 & Cellular senescence & $2.32 \mathrm{E}-12$ & 17 \\
\hline hsa05169 & Epstein-Barr virus infection & $9.58 \mathrm{E}-11$ & 17 \\
\hline hsa05166 & Human T-cell leukemia virus 1 infection & $3.39 \mathrm{E}-10$ & 17 \\
\hline hsa05165 & Human papillomavirus infection & $1.21 \mathrm{E}-07$ & 17 \\
\hline hsa05162 & Measles & $4.9 \mathrm{E}-12$ & 16 \\
\hline hsa05224 & Breast cancer & $1.08 \mathrm{E}-11$ & 16 \\
\hline & & & \\
\hline
\end{tabular}

\section{Drug-compound-target-pathway network}

In the drug-compound-target-pathway network, 4 red triangle nodes represented the drugs, while 16 green octagon nodes were on behalf of the compounds, 87 orange $V$-shape nodes were in the name of the potential targets, and the signaling pathways were represented by 20 purple diamond nodes. Altogether, 
there were 127 nodes in the network and 518 edges in the network, indicating that ZXF treated ICH with multiple compounds, multiple targets and multiple pathways.

As shown by topology analysis, the five compounds with the highest degree were Quercetin, betasitosterol, Mandenol, Myricanone, and aloe-emodin, suggesting that these five compounds may be the main compounds for ZXF against ICH. Also, the top five potential targets sorted by degree value were AKT1, RELA, MAPK1, TP53, TNF, indicating that these five targets may be the core targets in treating ICH by ZXF. In addition, the top five signaling pathways with the highest degree value are Fluid shear stress and atherosclerosis, AGE-RAGE signaling pathway in diabetic complications, PI3K-Akt signaling pathway, Kaposi sarcoma-associated herpesvirus infection, and Human cytomegalovirus infection, indicating that ZXF treated ICH mainly through these five signaling pathways.

\section{PPI network}

As was shown in Fig. 4(A), there were a total of 87 nodes and 1514 edges in the PPI network for potential targets. The average node degree was 34.8 . Sorted by the degree value, the top 10 potential targets, considered as core genes, were AKT1 (degree=68), TP53 (degree=68), and VEGFA (degree=68). =67), IL6 (degree=67), CASP3 (degree=66), JUN (degree=64), EGF (degree=64), PTGS2 (degree=63), EGFR (degree=61). Besides, it can be seen from Fig. 4(B) that 10 nodes and 45 edges were included in the PPI network of the core genes with the average node degree was 9 .

\section{Molecular docking}

As was shown in Table 4, the core targets (AKT1, MAPK1, RELA, TNF, TP53) and active compounds (betasitosterol, myricanone, aloe-emodin, quercetin, mandenol) obtained from drug-compound-target-pathway network were chosen to further verify compound-target interaction by molecular docking. In general, the lower the binding affinity was, the more effective the molecular docking was. And the binding affinity less than $5 \mathrm{kcal} / \mathrm{mol}$ would be regarded as effective molecular docking. According to the criteria, aloe-emodin, beta-sitosterol and quercetin would be three active compounds in ZXF. Additionally, MAPK1 and TNF may be therapeutic targets for ZXF against ICH. Furthermore, the hydrogen-bonding interaction between receptor (core target) and ligand (active compound) was shown in Fig. 6. 
Table 4

Molecular docking of core targets and active compounds

\begin{tabular}{|lllllll|}
\hline Target & PDB ID & \multicolumn{2}{l|}{ Affinity $\left(\mathrm{kcal}^{\prime} \mathrm{mol}^{-1}\right.$ ) } & & \\
\cline { 3 - 7 } & & Aloe_emodin & Beta_sitosterol & Mandenol & Myricanone & Quercetin \\
\hline AKT1 & 1UNQ & -6.6 & -7.4 & -4.3 & -4.2 & -6.4 \\
\hline MAPK1 & 4 IZ5 & -8.1 & -9.5 & -5.0 & -8.6 & -8.6 \\
\hline RELA & 1NFI & -8.3 & -7.6 & -4.8 & -7.1 & -8.4 \\
\hline TNF & 5 YOY & -8.2 & -9.0 & -5.5 & -9.0 & -8.4 \\
\hline TP53 & 3 Q05 & -9.0 & -7.8 & -4.9 & -8.2 & -9.1 \\
\hline
\end{tabular}

\section{Discussion}

$\mathrm{ICH}$ is a cerebrovascular disease with extremely high morbidity and mortality. Most patients are left with severe neurological impairment symptoms and relevant complications, which brings serious economic and psychological burdens to the society and families [34]. To make matters worse, there is yet no optimal therapy for $\mathrm{ICH}$, whether the surgical treatment or the conservative treatment [35]. Nowadays, more and more focus was fixed on the treatment of $\mathrm{ICH}$ in the field of traditional Chinese medicine [3638]. Previous study has shown that ZXF can improve the functional outcome of patients with ICH [11]. But the mechanism how the ZXF takes effect has not been extensively verified. This study aimed to explore the potential mechanism of ZXF against ICH through network pharmacology and molecular docking. And the results suggested that ZXF treated ICH through multiple compounds, multiple targets, and multiple pathways.

Through the search and screening of the TCMSP database, a total of 16 active compounds of ZXF were finally obtained. By constructing the drug-compound-target-pathway network, the top five active compounds sorted by degree value were Quercetin, beta-sitosterol, Mandenol, Myricanone, and aloeemodin. Quercetin has been proven to exert a variety of pharmacological effects related to cerebrovascular diseases, such as oxidative stress, atherosclerosis, ischemia-reperfusion injury, endothelial cell dysfunction [39]. Beta-sitosterol were proved to be effective in ICH mainly by reducing the risk of plasma cholesterol and atherosclerosis [40]. Several pharmacological properties on Mandenol have been reported including attenuating the expression of pro-inflammatory factors [41], Gudiya et al have shown that Myricanone was equipped with the biological functions of antioxidant, antiinflammatory, and anti-ischemia-reperfusion injury [42]. In addition, aloe-emodin is a widely existing anthraquinone in nature, which has been found to lower cholesterol by inhibiting PCSK9 [43].

Through the Uniprot database, OMIM database, Genecards database, and Drugbank database, there were 166 targets related to ZXF and 1258 targets related to ICH obtained in total. Among them, 87 targets 
shared by disease and drug altogether. In the drug-compound-target-pathway network, AKT1, MAPK1, RELA, TNF, and TP53 were the top five targets with the highest degree value, which were the core targets of ZXF against ICH. Huang et al. found that AKT1 can precisely regulate the synthesis of nitric oxide and further promote vascular remodeling [44]. In addition, AKT1 activation can promote the overexpression of glial cell-derived neurotrophic factor (GDNF) and thereby protect damaged neuronal cells [45]. Sun et al. indicated that RELA, a key transcription factor for hypoxic response, can promote the expression of CXCL12 under hypoxic conditions by combining with LINC01693 and thus promote hypoxia-induced angiogenesis [46]. MAPK1, belonging to the MAPK family, can be activated by LncRNA NEAT1 to enhance cell viability and inhibit cell apoptosis [47]. Wei et al. illustrated that the methylation of the TP53 promoter may be related to the occurrence of atherosclerosis, which has got wider audience in the research of atherosclerosis [48]. TNF has been shown to promote apoptosis of injured neurons by activating apoptosis signaling pathways, which has gained widespread attention in the field of neuroscience research [49].

According to the $\mathrm{GO}$ enrichment analysis, the function of potential targets were significantly enriched in a wealth of MFs, CCs and BPs. The receptor ligand activity, signaling receptor activator activity, and cytokine receptor binding were three MFs most closely related to treatment of ICH with ZXF. And the most important CCs involved in the ZXF against ICH were membrane raft, membrane microdomain, membrane region. Additionally, the response to lipopolysaccharide, response to molecule of bacterial origin, and cellular response to drug were the most significantly enriched BPs related to the treatment of ICH. KEGG enrichment analysis revealed that the overlapping targets of ZXF and ICH were mainly enriched in a number of signaling pathways, such as fluid shear stress and atherosclerosis, AGE-RAGE signaling pathway, PI3K-Akt signaling pathway, kaposi sarcoma-associated herpesvirus infection, and human cytomegalovirus infection. Fluid shear stress and atherosclerosis plays a vital role in maintaining cardiovascular and cerebrovascular homeostasis by keeping the balance of redox and thus can protect vascular endothelial cells from oxidative stress [50]. AGE-RAGE signaling pathway has been proved to play a role as a pro-inflammatory mediator, which can activate the transcription factor NF-Kb and trigger the inflammation and oxidative stress [51]. Luo et al suggested that PI3K-Akt signaling pathway can reduce the level of reactive oxygen species and lipid deposition, which can inhibit the formation of plaques and reverse the progress of atherosclerosis [52]. Sun et al illustrated that activating the PI3K-Akt signaling pathway can up-regulate the expression of MIAT and aggravate atherosclerotic injury [53]. Kaposi sarcoma-associated herpesvirus infection signaling pathway is mainly related to the secretion of pro-angiogenic cytokines and pro-inflammatory factors, which is able to promote angiogenesis and induce inflammation signals [54]. Human cytomegalovirus infection signaling pathway plays a vital role in controlling the differentiation, proliferation, migration, and angiogenesis of cells and production and function of cytokines [55].

In the present study, network pharmacology was used to discover the active compounds, potential targets, and signaling pathways of ZXF against ICH to clarify the possible mechanism of the treatment. Additionally, the molecular docking was utilized to further verify the interaction between the compounds and the targets. Moreover, ZXF was picked out based on the preliminary clinical research of our team, 
which was much more reliable than those unproven drugs. However, the main limitations of our study needed to be acknowledged. This study was based on the data in the existing literature and the compatibility of traditional Chinese herbs in clinical practice has not been taken into consideration. Thus, more multicenter prospective randomized clinical trials and better basic researches will be needed to validate the effect in the future.

\section{Conclusion}

All in all, the study expounded that ZXF could exert pharmacological effects through promoting angiogenesis, anti-inflammatory, anti-oxidative stress, and reversing atherosclerosis, which played a vital role in the treatment of $\mathrm{ICH}$. Both network pharmacology and molecular docking were used to reveal the underlying mechanism of the treatment, which provided theoretical guidance for the subsequent in-depth basic researches. Nevertheless, a large number of clinical trials and basic researches were still ought to be carried out to confirm these results.

\section{Abbreviations}

ZXF: Zhongfeng Xingnao Formula;

$\mathrm{ICH}$ : intracerebral hemorrhage;

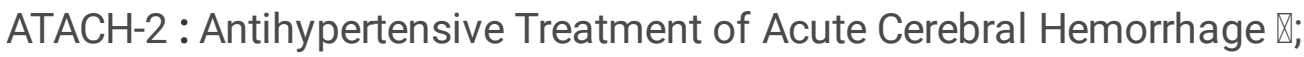

i-DEF: Intracerebral Haemorrhage Deferoxamine;

TICH-2: Tranexamic acid for hyperacute primary IntraCerebral Haemorrhage;

TCM: traditional Chinese medicine;

TCMSP: Traditional Chinese Medicine Systems Pharmacology Database and Analysis Platform; DH: rhubarb;

SQ: sanchi;

CX: szechwan lovage rhizome;

HS: red ginseng;

OB: oral bioavailability;

DL: drug-likeness;

IEP: intestinal epithelial permeability;

BBB: blood-brain-barrier; 
UniProtKB: UniProt Knowledge Base;

GO: Gene Ontology;

KEGG: Kyoto Encyclopedia of Genes and Genomes;

BP: biological process;

CC: cell component;

MF: molecular function;

PPI: protein-protein interaction

\section{Declarations}

\section{Ethics approval and consent to participate:}

Not applicable.

\section{Consent for publication:}

Not applicable.

\section{Availability of data and materials:}

All data are available in the manuscript and they are showed in figures and tables

\section{Competing interests:}

The authors declare that they have no competing interests.

\section{Funding】}

This work was supported by National Key R\&D Program of China (2018YFC1705002) and Key-Area Research and Development Program of Guangdong Province (2020B1111100009).

\section{Authors' contributions $\rrbracket$}

Jian-wen Guo and Ye-feng Cai conceived and designed the research; Can Wan and Guang-yao Zhang collected and analyzed the data; Zi-yi Zhou and Yun Lu provided useful suggestions on the methodology; 
Can Wan wrote the paper. All authors read and approved the final version of the manuscript.

\section{Acknowledgements $\llbracket$}

The authors would like to thank the reviewers and also the authors of all references. The reviewer's advice really makes the great improvement of this paper.

\section{References}

1. Wang YJ, Gu HQ, Zhai Y. China Stroke Statistics 2019: A Report From the National Center for Healthcare Quality Management in Neurological Diseases, China National Clinical Research Center for Neurological Diseases, the Chinese Stroke Association, National Center for Chronic and Noncommunicable Disease Control and Prevention, Chinese Center for Disease Control and Prevention and Institute for Global Neuroscience and Stroke Collaborations. Stroke \& Vascular Neurology 2020;5(3):1-29.doi:10.1136/svn-2020000457

2. Otite FO, Khandelwal P, Malik AM, et al. Ten-Year Temporal Trends in Medical Complications After Acute Intracerebral Hemorrhage in the United States. Stroke. 2017;48(3):596603.doi:10.1161/strokeaha.116.015746

3. Writing Group M, Mozaffarian D, Benjamin EJ, et al. Heart Disease and Stroke Statistics-2016 Update: A Report From the American Heart Association. Circulation. 2016;133(4):e38360.doi:10.1161/CIR.0000000000000350

4. Hemphill JC, Greenberg SM, Anderson CS, et al. Guidelines for the Management of Spontaneous Intracerebral Hemorrhage: A Guideline for Healthcare Professionals From the American Heart Association/American Stroke Association. Stroke. 2015;46(7):203260.doi:10.1161/STR.0000000000000069

5. Qureshi Al, Palesch YY, Barsan WG, et al. Intensive Blood-Pressure Lowering in Patients with Acute Cerebral Hemorrhage. N Engl J Med. 2016;375(11):1033-43.doi:10.1056/NEJMoa1603460

6. Selim M, Foster LD, Moy CS, et al. Deferoxamine mesylate in patients with intracerebral haemorrhage (i-DEF): a multicentre, randomised, placebo-controlled, double-blind phase 2 trial. The Lancet Neurology. 2019;18(5):428-38.doi:10.1016/s1474-4422(19)30069-9

7. Sprigg N, Flaherty K, Appleton JP, et al. Tranexamic acid for hyperacute primary IntraCerebral Haemorrhage ( $\mathrm{TICH}-2)$ : an international randomised, placebo-controlled, phase 3 superiority trial. The Lancet. 2018;391(10135):2107-15.doi:10.1016/s0140-6736(18)31033-x

8. Xu S, Pang Q, Lin Z, et al. Effect of integrated traditional Chinese and Western medicine therapy for acute hypertensive intracerebral hemorrhage: a meta-analysis. Artif Cells Nanomed Biotechnol. 2017;45(6):1-6.doi:10.1080/21691401.2016.1215327

9. Song J, Lyu Y, Wang P, et al. Treatment of Naoxueshu Promotes Improvement of Hematoma Absorption and Neurological Function in Acute Intracerebral Hemorrhage Patients. Front Physiol. 
2018;9:933.doi:10.3389/fphys.2018.00933

10. Li P, Tang T, Liu T, et al. Systematic Analysis of tRNA-Derived Small RNAs Reveals Novel Potential Therapeutic Targets of Traditional Chinese Medicine (Buyang-Huanwu-Decoction) on Intracerebral Hemorrhage. Int J Biol Sci. 2019;15(4):895-908.doi:10.7150/ijbs.29744

11. Zhang XY, Jin W, Chen SH. Clinical Observation on primary-Qi-tonifying and Brain-resuscitating Therapy for Treating Acute Cerebral Hemorrhage. Liaoning Journal of Traditional Chinese Medicine. 2012;39(6):968-71.doi:10.13192/j.ljtcm.2012.06.15.zhangxy.003

12. Zhang R, Zhu X, Bai H, et al. Network Pharmacology Databases for Traditional Chinese Medicine: Review and Assessment. Front Pharmacol. 2019;10:123.doi:10.3389/fphar.2019.00123

13. Hopkins AL. Network pharmacology. NATURE BIOTECHNOLOGY. 2007;25(10):1110-1

14. Lee WY, Lee CY, Kim YS, et al. The Methodological Trends of Traditional Herbal Medicine Employing Network Pharmacology. Biomolecules. 2019;9(8).doi:10.3390/biom9080362

15. Pinzi L, Rastelli G. Molecular Docking: Shifting Paradigms in Drug Discovery. Int J Mol Sci. 2019;20(18).doi:10.3390/ijms20184331

16. Ru JL LP, Wang JN, Zhou W. TCMSP: a database of systems pharmacology for drug discovery from herbal medicines. Journal of Cheminformatics. 2014;6(13):1-6

17. Kim MT, Sedykh A, Chakravarti SK, et al. Critical evaluation of human oral bioavailability for pharmaceutical drugs by using various cheminformatics approaches. Pharm Res. 2014;31(4):100214.doi:10.1007/s11095-013-1222-1

18. SMY TS, Li YY, Li Q. Drug-likeness analysis of traditional Chinese medicines: 1. property distributions of drug-like compounds, non drug-like compounds and natural compounds from traditional Chinese medicines. Journal of Cheminformatics. 2012

19. UniProt C. UniProt: a worldwide hub of protein knowledge. Nucleic Acids Res. 2019;47(D1):D506D15.doi:10.1093/nar/gky1049

20. Amberger JS, Hamosh A. Searching Online Mendelian Inheritance in Man (OMIM): A Knowledgebase of Human Genes and Genetic Phenotypes. Curr Protoc Bioinformatics. 2017;58:1 2 1- 2 12.doi:10.1002/cpbi.27

21. Michael R VC, Jaime P, Doron L. GeneCards: a novel functional genomics compendium with automated data mining and query reformulation support. Bioinformatics. 1998;14(8):656-64

22. Wishart DS, Feunang YD, Guo AC, et al. DrugBank 5.0: a major update to the DrugBank database for 2018. Nucleic Acids Res. 2018;46(D1):D1074-D82.doi:10.1093/nar/gkx1037

23. Yu G, Wang LG, Han Y, et al. clusterProfiler: an R package for comparing biological themes among gene clusters. OMICS. 2012;16(5):284-7.doi:10.1089/omi.2011.0118

24. Yu G, Wang LG, Yan GR, et al. DOSE: an R/Bioconductor package for disease ontology semantic and enrichment analysis. Bioinformatics. 2015;31(4):608-9.doi:10.1093/bioinformatics/btu684

25. Ashburner M, Ball CA, Blake JA, et al. Gene ontology: tool for the unification of biology. The Gene Ontology Consortium. Nat Genet. 2000;25(1):25-9.doi:10.1038/75556 
26. Joanne W DK. The Kyoto Encyclopedia of Genes and Genomes-KEGG. Yeast. 2000;17:48-55

27. Paul S AM, Owen O, Nitin SB. Cytoscape: A Software Environment for Integrated Models of Biomolecular Interaction Networks. Genome Research. 2003;13:2498-504.doi:10.1101/

28. Szklarczyk D, Morris JH, Cook H, et al. The STRING database in 2017: quality-controlled proteinprotein association networks, made broadly accessible. Nucleic Acids Res. 2017;45(D1):D362D8.doi:10.1093/nar/gkw937

29. Ferreira LG, Dos Santos RN, Oliva G, et al. Molecular docking and structure-based drug design strategies. Molecules. 2015;20(7):13384-421.doi:10.3390/molecules200713384

30. Burley SK, Berman HM, Bhikadiya C, et al. RCSB Protein Data Bank: biological macromolecular structures enabling research and education in fundamental biology, biomedicine, biotechnology and energy. Nucleic Acids Research. 2019;47(D1):D464-D74.doi:10.1093/nar/gky1004

31. Morris GM, Huey R, Lindstrom W, et al. AutoDock4 and AutoDockTools4: Automated docking with selective receptor flexibility. J Comput Chem. 2009;30(16):2785-91.doi:10.1002/jcc.21256

32. Di Muzio E, Toti D, Polticelli F. DockingApp: a user friendly interface for facilitated docking simulations with AutoDock Vina. J Comput Aided Mol Des. 2017;31(2):213-8.doi:10.1007/s10822016-0006-1

33. Lill MA, Danielson ML. Computer-aided drug design platform using PyMOL. Journal of ComputerAided Molecular Design. 2010;25(1):13-9.doi:10.1007/s10822-010-9395-8

34. Feigin VL, Roth GA, Naghavi M, et al. Global burden of stroke and risk factors in 188 countries, during 1990-2013: a systematic analysis for the Global Burden of Disease Study 2013. The Lancet Neurology. 2016;15(9):913-24.doi:10.1016/s1474-4422(16)30073-4

35. Sembill JA, Huttner HB, Kuramatsu JB. Impact of Recent Studies for the Treatment of Intracerebral Hemorrhage. Current Neurology and Neuroscience Reports. 2018;18(10).doi:10.1007/s11910-0180872-0

36. Zeng L, Guo J, Wang J, et al. Clinical re-evaluation of removing blood stasis therapy in treating acute intracerebral hemorrhage safety and efficacy: a protocol for a randomized, controlled, multicenter study (CRRICH Trial). Springerplus. 2016;5(1):1466.doi:10.1186/s40064-016-3136-y

37. Zhang Q, Zeng L, Chen $X$, et al. Clinical Evaluation of Herbal Medicine (ICH-012) in Treating Acute Cerebral Haemorrhage: Safety and Efficacy from 6- to 72-Hour Time Window (CRRICHTrial-II). Evidence-Based Complementary and Alternative Medicine. 2018;2018:17.doi:10.1155/2018/3120179

38. Zeng LL TG, Wang J, Zhong JB. Safety and efficacy of herbal medicine for acute intracerebral hemorrhage (CRRICH): a multicentre randomised controlled trial. BMJ Open. 2019;9:1-9.doi:10.1136/

39. Patel RV, Mistry BM, Shinde SK, et al. Therapeutic potential of quercetin as a cardiovascular agent. Eur J Med Chem. 2018;155:889-904.doi:10.1016/j.ejmech.2018.06.053

40. Ong SL, Mah SH, Lai HY. Porcine Pancreatic Lipase Inhibitory Agent Isolated from Medicinal Herb and Inhibition Kinetics of Extracts from Eleusine indica (L.) Gaertner. J Pharm (Cairo). 2016;2016:8764274.doi:10.1155/2016/8764274 
41. Park SY, Seetharaman R, Ko MJ, et al. Ethyl linoleate from garlic attenuates lipopolysaccharideinduced pro-inflammatory cytokine production by inducing heme oxygenase-1 in RAW264.7 cells. Int Immunopharmacol. 2014;19(2):253-61.doi:10.1016/j.intimp.2014.01.017

42. Gupta G, Siddiqui MA, Khan MM, et al. Current Pharmacological Trends on Myricetin. Drug Research. 2020;70(10):448-54.doi:10.1055/a-1224-3625

43. Su ZL, Hang PZ, Hu J, et al. Aloe-emodin exerts cholesterol-lowering effects by inhibiting proprotein convertase subtilisin/kexin type 9 in hyperlipidemic rats. Acta Pharmacol Sin. 2020;41(8):108592.doi:10.1038/s41401-020-0392-8

44. Huang J, Cai C, Zheng T, et al. Endothelial Scaffolding Protein ENH (Enigma Homolog Protein) Promotes PHLPP2 (Pleckstrin Homology Domain and Leucine-Rich Repeat Protein Phosphatase 2)Mediated Dephosphorylation of AKT1 and eNOS (Endothelial NO Synthase) Promoting Vascular Remodeling. Arterioscler Thromb Vasc Biol. 2020;40(7):1705-21.doi:10.1161/ATVBAHA.120.314172

45. Gao J, Kang XY, Sun S, et al. MES23.5 DA Immortalized Neuroblastoma Cells Self-protect Against Early Injury by Overexpressing Glial Cell-derived Neurotrophic Factor via Akt1/Eya1/Six2 Signaling. J Mol Neurosci. 2020;70(3):328-39.doi:10.1007/s12031-019-01416-7

46. Sun $Y$, Xiong $X$, Wang $X$. RELA promotes hypoxia-induced angiogenesis in human umbilical vascular endothelial cells via LINC01693/miR-302d/CXCL12 axis. J Cell Biochem. 2019;120(8):1254958.doi:10.1002/jcb.28521

47. Zhang H, Ji N, Gong X, et al. NEAT1/miR-140-3p/MAPK1 mediates the viability and survival of coronary endothelial cells and affects coronary atherosclerotic heart disease. Acta Biochim Biophys Sin (Shanghai). 2020;52(9):967-74.doi:10.1093/abbs/gmaa087

48. Wei Y, Sun Z, Wang Y, et al. Methylation in the TP53 promoter is associated with ischemic stroke. Mol Med Rep. 2019;20(2):1404-10.doi:10.3892/mmr.2019.10348

49. Shao $X$, Yang $X$, Shen J, et al. TNF-alpha-induced p53 activation induces apoptosis in neurological injury. J Cell Mol Med. 2020;24(12):6796-803.doi:10.1111/jcmm.15333

50. McSweeney SR, Warabi E, Siow RCM. Nrf2 as an Endothelial Mechanosensitive Transcription Factor. Hypertension. 2016;67(1):20-9.doi:10.1161/hypertensionaha.115.06146

51. Roy B. Biomolecular basis of the role of diabetes mellitus in osteoporosis and bone fractures. World J Diabetes. 2013;4(4):101-13.doi:10.4239/wjd.v4.i4.101

52. Luo Y SG, Dong X, Wang M, Qin M. Isorhamnetin Attenuates Atherosclerosis by Inhibiting Macrophage Apoptosis via PI3K/AKT Activation and HO-1 Induction. Plos One. 2015;10(3):119.doi:10.1371/journal.pone.0120259.g001

53. Sun G, Li Y, Ji Z. Up-regulation of MIAT aggravates the atherosclerotic damage in atherosclerosis mice through the activation of PI3K/Akt signaling pathway. Drug Deliv. 2019;26(1):6419.doi:10.1080/10717544.2019.1628116

54. Schulz TF, Cesarman E. Kaposi Sarcoma-associated Herpesvirus: mechanisms of oncogenesis. Curr Opin Virol. 2015;14:116-28.doi:10.1016/j.coviro.2015.08.016 
55. Murray MJ, Peters NE, Reeves MB. Navigating the Host Cell Response during Entry into Sites of Latent Cytomegalovirus Infection. Pathogens. 2018;7(1): 1-18.doi:10.3390/pathogens7010030

\section{Figures}

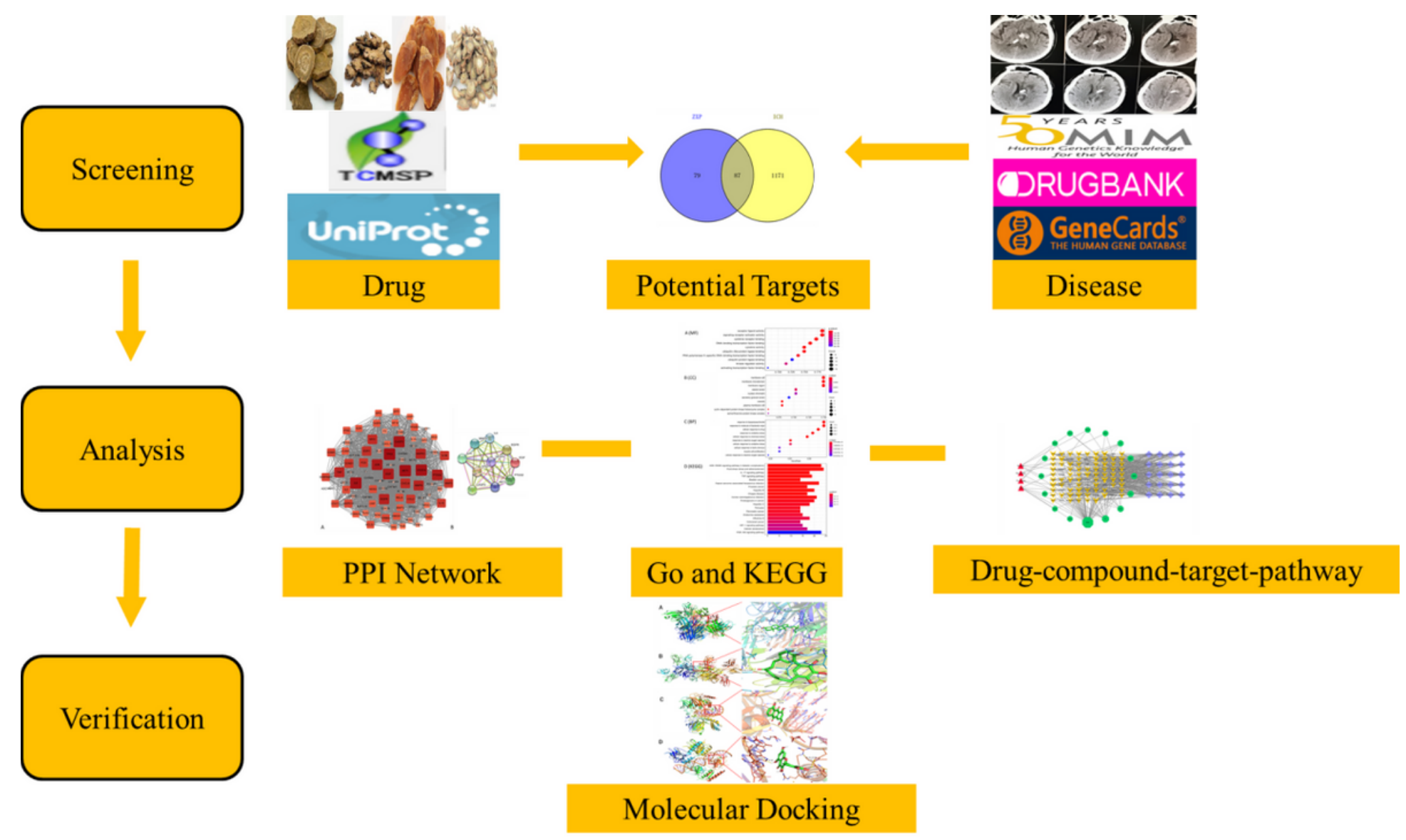

Figure 1

The sketch map for potential mechanism of Zhongfeng Xingnao Formula against intracerebral hemorrhage 


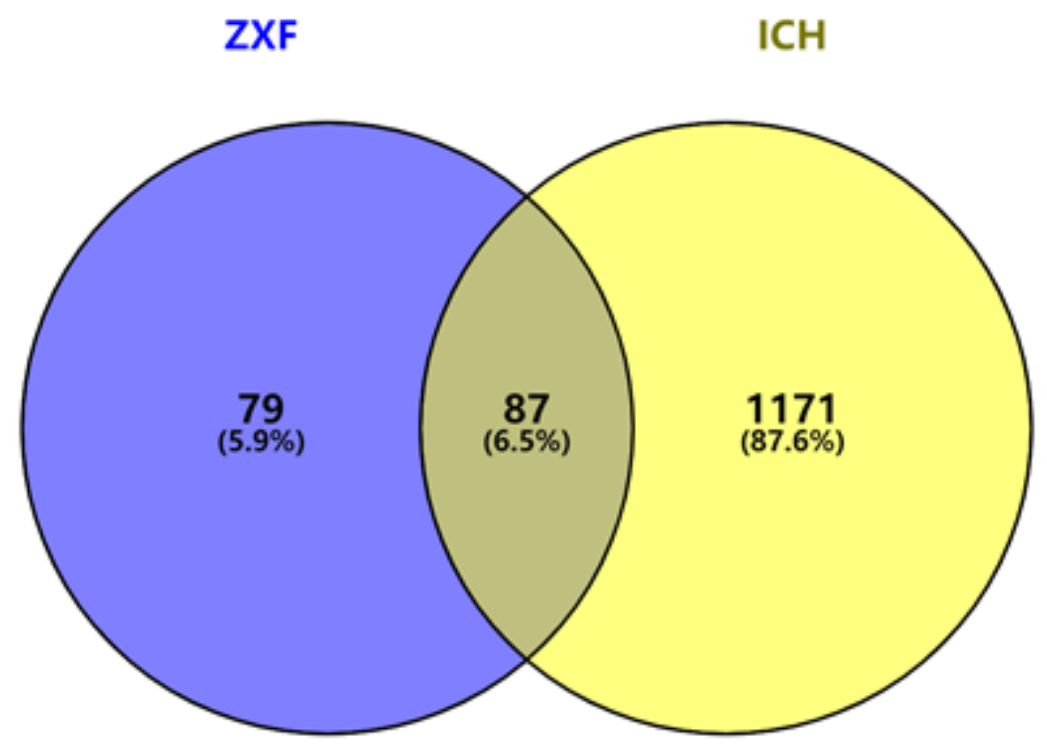

Figure 2

Venn diagram of overlapping targets of Zhongfeng Xingnao Formula (ZXF) and intracerebral hemorrhage $(\mathrm{ICH})$ 


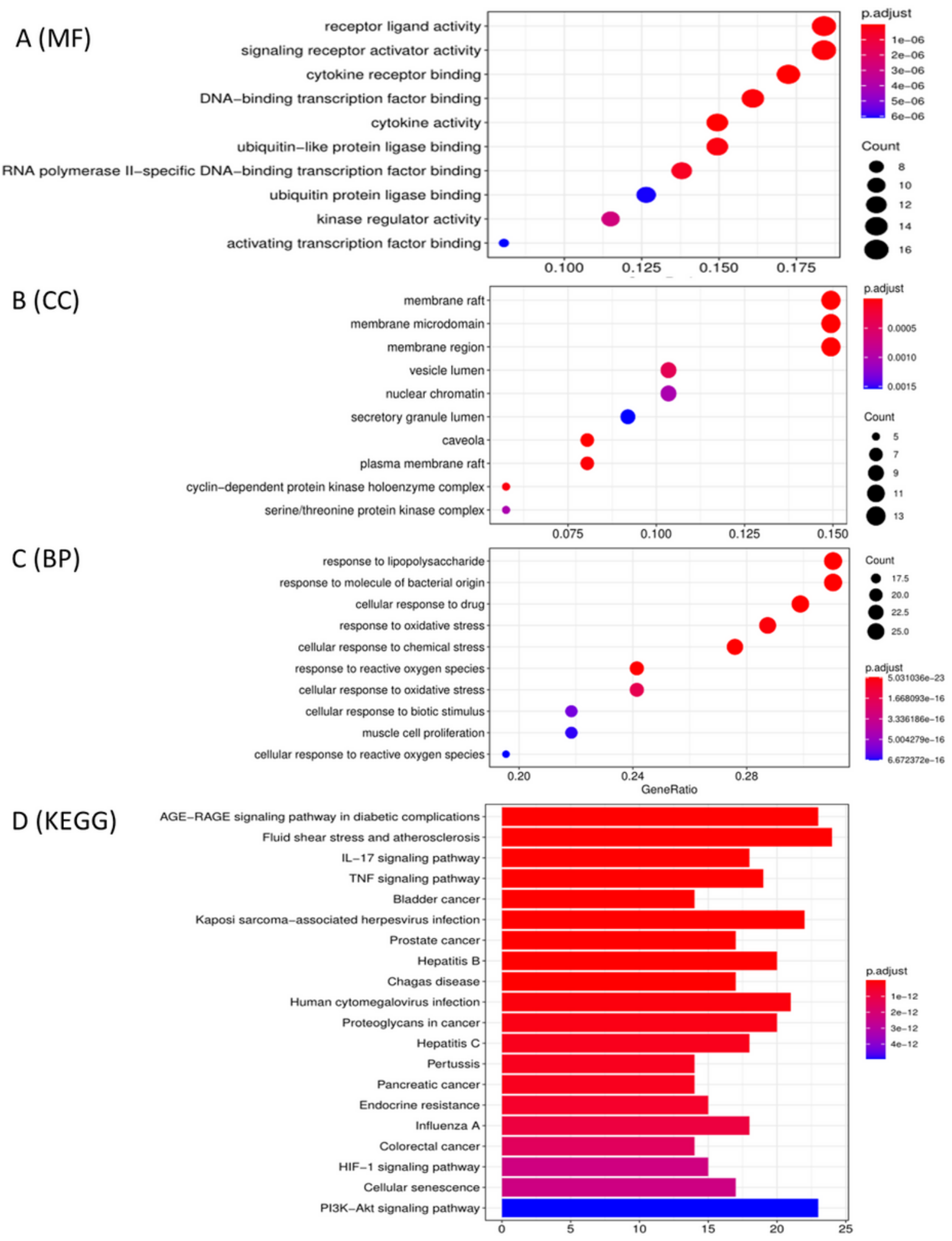

Figure 3

GO and KEGG enrichment analysis. (A) (B) (C) Bubble plots of GO enrichment analysis. The size of the bubble represented the number of enriched potential targets. The color of the bubble indicated the significance of enrichment. (D) Bar plots of KEGG enrichment analysis. The length of the bar showed the number of enriched potential genes. The color of the bar suggested the significance of the pathway. 


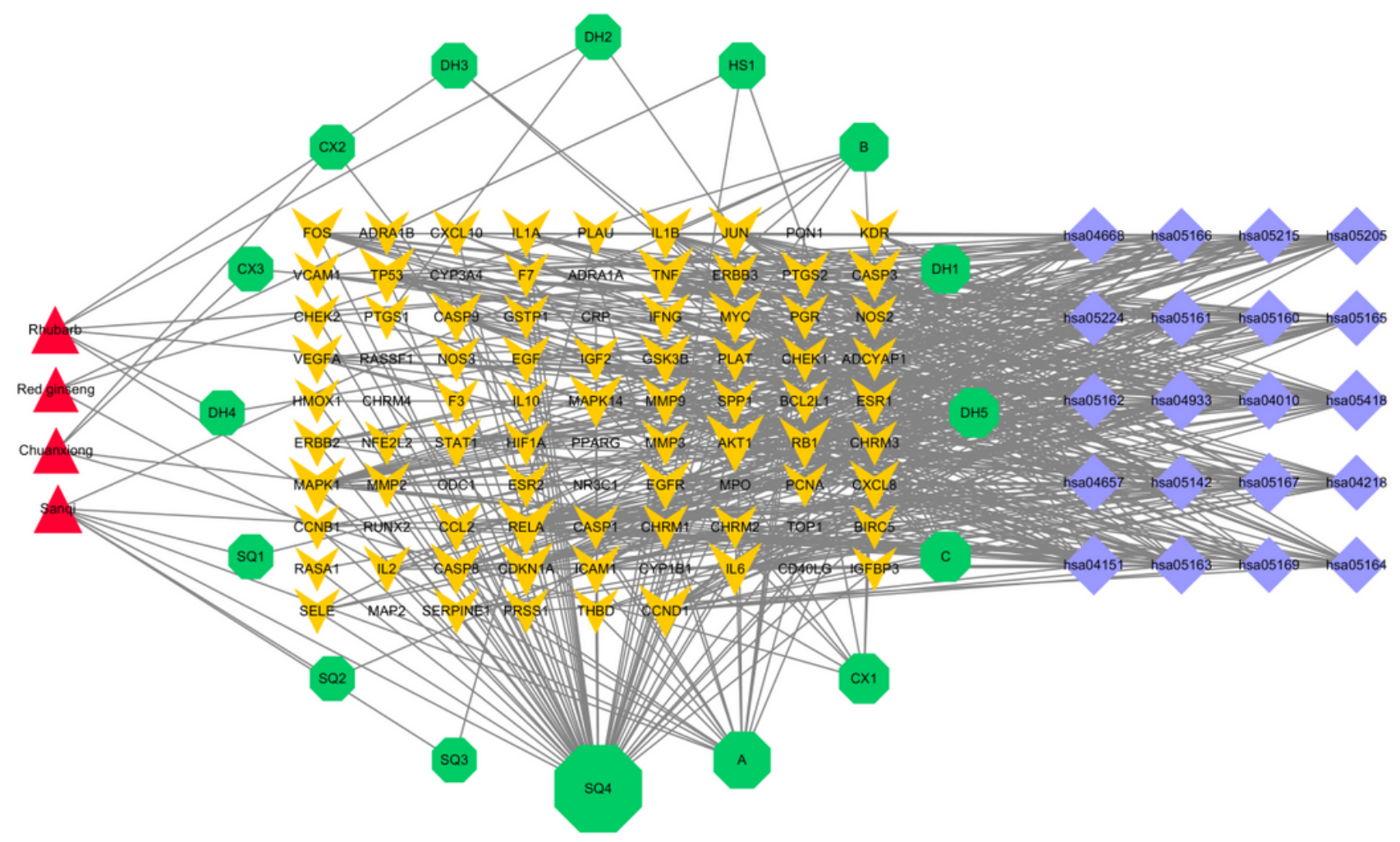

Figure 4

Drug-compound-target-pathway network. Four drugs, sixteen compounds, eighty seven potential targets, twenty signaling pathways were included with the size of nodes represented the degree value. 


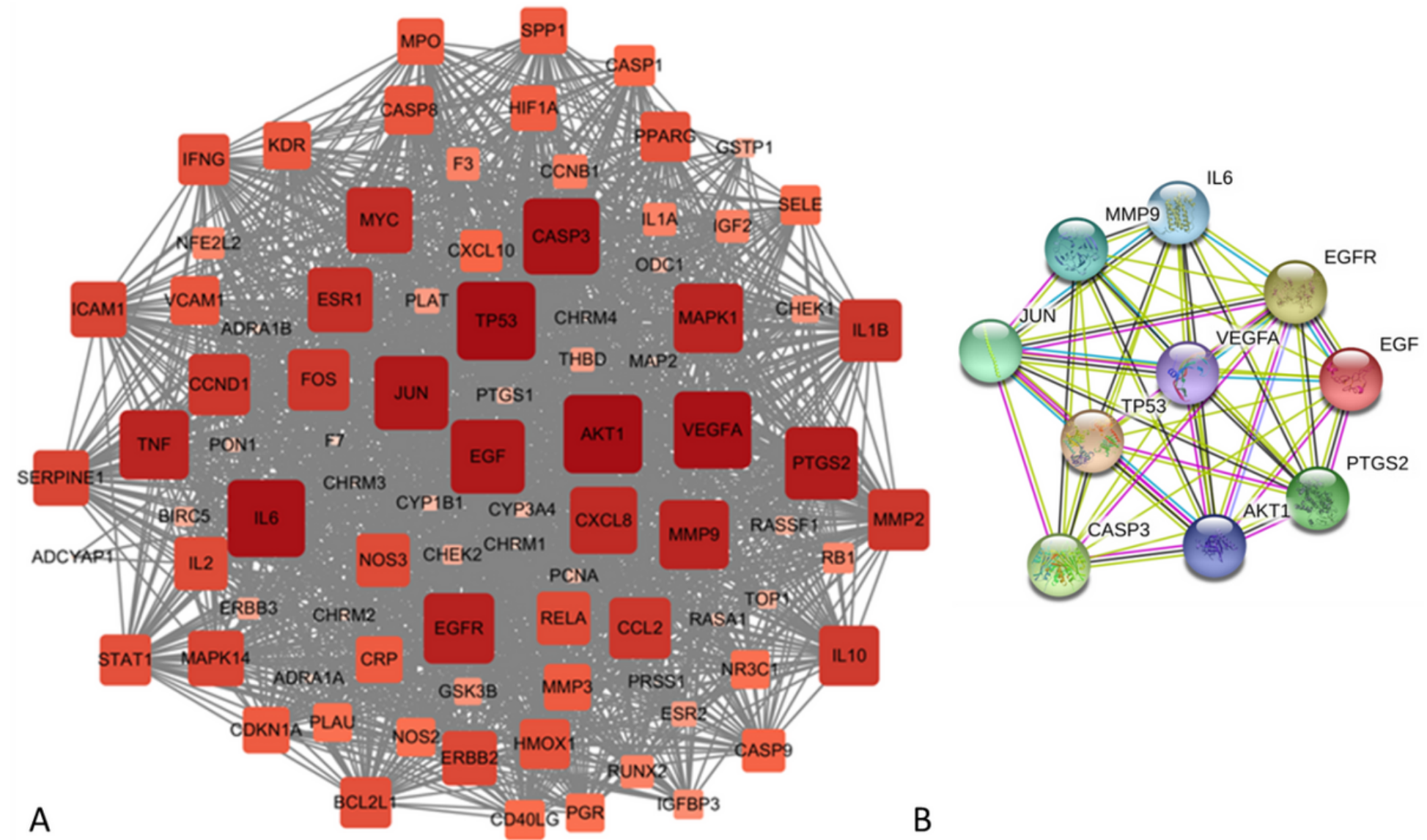

\section{Figure 5}

PPI network of potential targets (A) and core genes (B) for Zhongfeng Xingnao Formula against intracerebral hemorrhage 

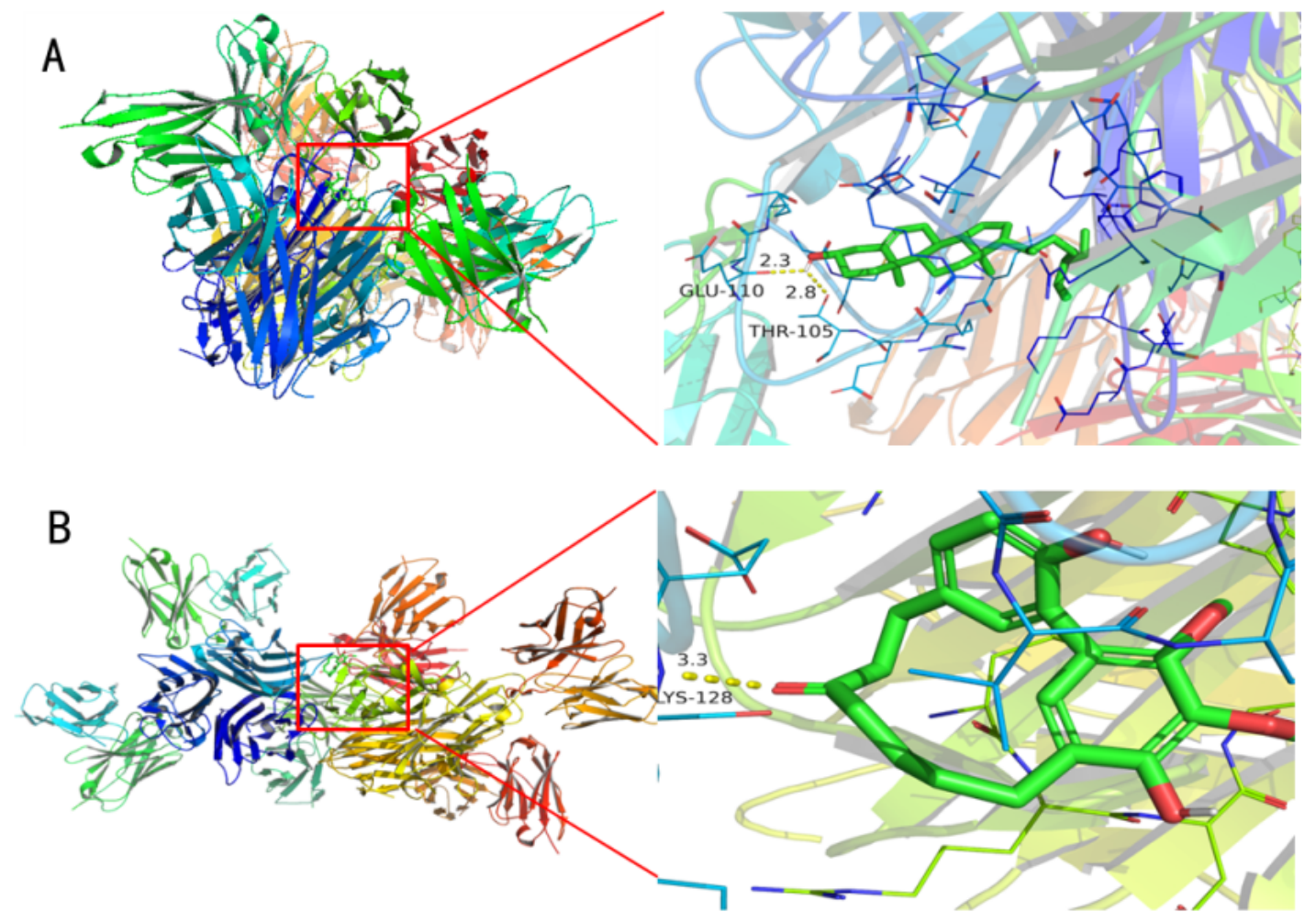

C
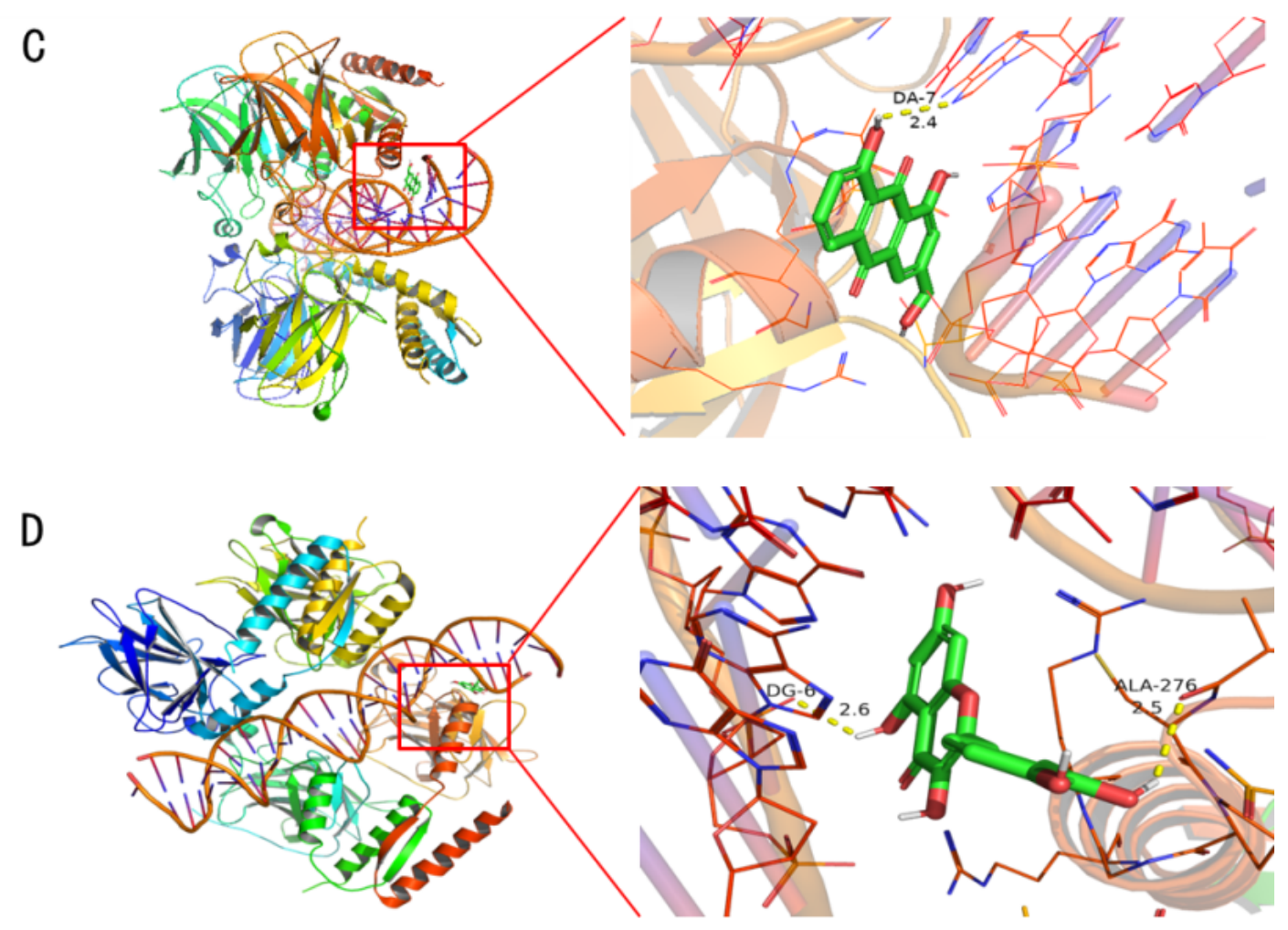

Figure 6

Molecular docking of core targets and active compounds. (A) TNF and beta-sitosterol. (B) TNF and myricanone. (C) TP53 and aloe-emodin. (D) TP53 and quercetin. 